\title{
DESIGN OF A TEACHING UNIT TO DEVELOP PRIMARY SCHOOL STUDENTS' REASONING ABOUT UNCERTAINTY IN MULTI-STEP CHANCE EXPERIMENTS
}

\author{
Daniel Frischemeier \& Rolf Biehler \\ University of Paderborn, Germany \\ dafr@,math.upb.de
}

\begin{abstract}
Statistical reasoning and the confrontation with first ideas of uncertainty can already be enhanced in primary school. A challenge is how to relate theoretical-combinatorial aspects to empirical frequency aspects, given that fraction concepts are usually not available at primary school. In the frame of a Design Based Research approach we have designed and realized a teaching sequence consisting of seven lessons to develop statistical reasoning about uncertainty of grade 4 students (age 10-11). To supervise their learning processes we collected data on different levels: (a) written pre/post-tests, (b) working notes after each lesson and (c) interviews after the teaching unit. In this paper we will mainly present the design of teaching unit and first results from the analysis of pre-and posttests.
\end{abstract}

\section{INTRODUCTION}

Since establishing the leading idea of data, frequency and chance in the German primary school curriculum (Hasemann \& Mirwald, 2012), statistics has received increased attention. Recommended activities from a perspective of understanding uncertainty are -amongst others- the interpretation and comparison of probabilities of certain events in one- or multi-step chance experiments. Statistical reasoning and the confrontation with first ideas of uncertainty can and should already be enhanced in primary school. Practical examples for enhancing statistical reasoning in primary school can be found in Leavy, Meletiou-Mavrotheris, and Paparistodemou (2018). In the aspect of reasoning about uncertainty Batanero (2015) distinguishes between three conceptualizations of randomness: (i) Randomness as equiprobability, (ii) randomness as stability of frequencies and (iii) subjective view of randomness. In (i) probability is defined as "the number of favorable cases to a particular event divided by the number of all cases possible in that experiment, provided all the possible cases are equiprobable" (Batanero, 2015, p. 36). The second concept (ii) is related to the empirical law of large numbers, probability is defined according to Batanero $(2015$, p. 37) as "the hypothetical number towards which the relative frequency tends". The third concept (iii) of subjective view "considered probability as a personal degree of belief that depends on a person's knowledge or experience" (Batanero, 2015, p. 37). The main aim of this paper is to describe the design of a teaching unit which covers each of the three conceptualizations of randomness to develop primary school students reasoning about uncertainty. The second aim of this paper is to evaluate in which way the teaching unit offers a contribution to the development of reasoning of primary school students about uncertainty in multi-step chance experiments.

\section{A TEACHING UNIT TO ENHANCE STATISTICAL REASONING IN PRIMARY SCHOOL}

There are several examples that even the interpretation of probabilities of events in multi-step chance experiments can already be covered in primary school (see for example Weustenfeld, 2007 and Jackes-Schulte, 2016). In the frame of the master thesis of Plückebaum (2018) using the Design-based research approach (Cobb, Confrey, diSessa, Lehrer, \& Schauble, 2003) we have designed and realized a teaching sequence consisting of seven lessons taking into account all three conceptualizations of randomness of Batanero (2015) to develop the reasoning about uncertainty of grade 4 students. The teaching unit is designed on the principles of SRLE (Garfield \& Ben-Zvi, 2008) and one fundamental design idea is the use of digital tools like TinkerPlots (Konold \& Miller, 2011) which can support modelling multi-step chance experiments. The teaching unit consists of seven lessons (45 minutes each) and has been conducted in a fourth grade at a primary school in a rural area in Germany. The pupils had no specific pre-knowledge in statistics apart from collecting data in tallies and creating and reading bar charts and reading pie charts. For an overview on the lessons see Table 1. In the first lesson the pupils are introduced into statistical diagrams like bar charts and pie charts. They learn how to create bar charts and then in a next step the teacher discusses with them how to read and interpret bar charts and pie charts. In the second lesson the students were confronted with several terminologies of probability like "certain", "probable", "unlikely" and "impossible". 
Table 1. Overview of the contents series of lessons

\begin{tabular}{cl}
\hline No & Content of lesson \\
\hline $\mathbf{1}$ & Pupils learn about how to read and interpret statistical bar graphs and pie charts \\
$\mathbf{2}$ & $\begin{array}{l}\text { Pupils estimate and compare probabilities of events by classifying them in "certain", } \\
\text { "possible", "unlikely" and "impossible". }\end{array}$ \\
$\mathbf{3}$ & $\begin{array}{l}\text { Pupils conduct hands-on experiment "throw of a dice" and collect data to get the insight that } \\
\text { the probability for each side of the dice is equal. }\end{array}$ \\
$\mathbf{4}$ & $\begin{array}{l}\text { Pupils are introduced in the sampler of TinkerPlots and get first experiences of the empirical } \\
\text { law of large numbers by simulating the throw of two coins with the TinkerPlots sampler }\end{array}$ \\
$\mathbf{5}$ & $\begin{array}{l}\text { Pupils conduct hands-on experiment "throw of two dice", collect the sum of the two dice for } \\
\text { each throw and collect data to use the frequencies of occurrence of the different sums to } \\
\text { make statements with regard to the probability of the events of different }\end{array}$ \\
$\mathbf{6 -}$ & $\begin{array}{l}\text { Pupils conduct TinkerPlots simulation "throw of two dice" and collect data to compare the } \\
\text { probability of events. Pupils try to find explanations why some sums appear more frequent } \\
\text { than other sums when throwing two dice. }\end{array}$ \\
\hline
\end{tabular}

Together with the teacher the students evaluate events of several chance experiments using the expressions "certain", "probable", "unlikely" and "impossible". As accompanying material the teacher offers a strip with a scale from "impossible" to "certain" which the students use to show and to visualize their expectation. In a next step the teacher expands the scale and adds frequency descriptions like never, seldom, often and always for each terminology ("impossible", etc., see Figure $1)$.

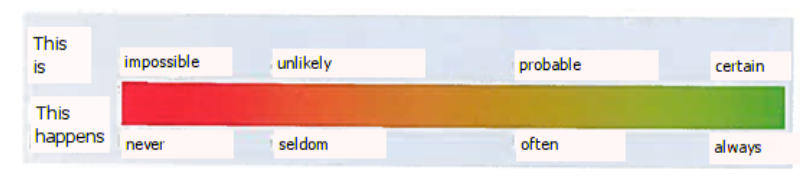

Figure 1: Scales for indicating the probabilities

The aim of the third lesson is that the students recognize that each side of a fair dice is equiprobable. So the students work in pairs and are asked in hands-on activities to throw a dice fifty times and to document the frequencies of the occurrences of each side. The teacher then collects the data from all peers on the board in front of the class and calculates the frequencies of the occurrences for each side as total. Collecting the data of all ten participating groups the pupils can see that the absolute frequencies of each side are close together. Since it is not possible to use percentages in primary school, the teacher uses notations like "In 79 of 500 cases the side 1 has occurred" or "in 78 of 500 cases the side 2 has occurred" to make the frequencies comparable and to get the expectation that each side is equiprobable. In the fourth lesson the pupils are introduced in the simulation of chance experiments with the TinkerPlots sampler. At first the teacher demonstrates how to realize this experiment in the TinkerPlots sampler (see Figure 2).
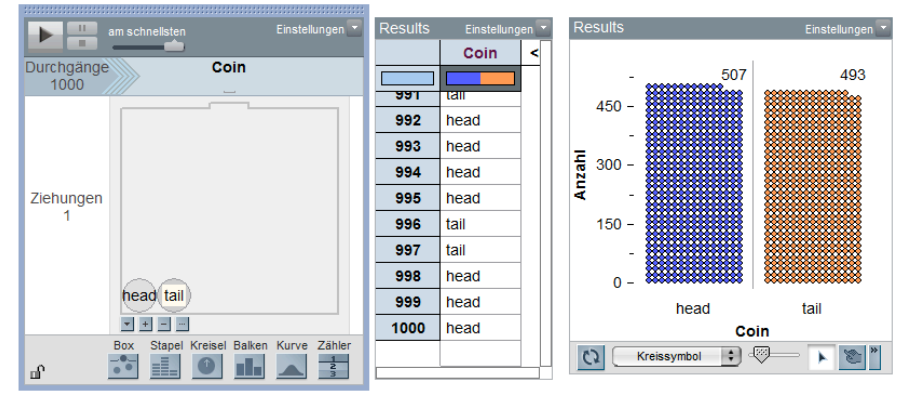

Figure 2: TinkerPlots sampler with results of the chance experiment „throw of two coins“ 
He demonstrates the creation of a box with the balls labelled "head" and "tail" symbolizing a fair coin and the teacher runs this sampler 1000 times to collect data. Then he creates a plot to display the distribution of "heads" and "tails". By running the sampler several times the pupils get the impression that the absolute frequencies of " $\mathrm{H}$ " and " $\mathrm{T}$ " are in most of the times close together. For instance, in figure 2, the proportion of head (" $\mathrm{H}$ ") and tail ("T") is 507:493. As introductory example for a multi-step chance experiment the teacher chooses the throw of two coins. This example was realized in a very short way, the introduction into multi-step chance experiments was continued in lesson 5. In the fifth lesson the teacher chooses a more complex chance experiment ("sum of two dice") to tackle the aspect of modelling multi-step chance experiments. The task for the pupils was to find out which sum is more likely to appear when throwing two dice. The pupils are paired in groups and each group was given two dice and the task to throw the two dice fifty times and to document their outcomes. Then the teacher collects the results of all ten groups (see the realization on the board in Figure 3) to make several explorations.

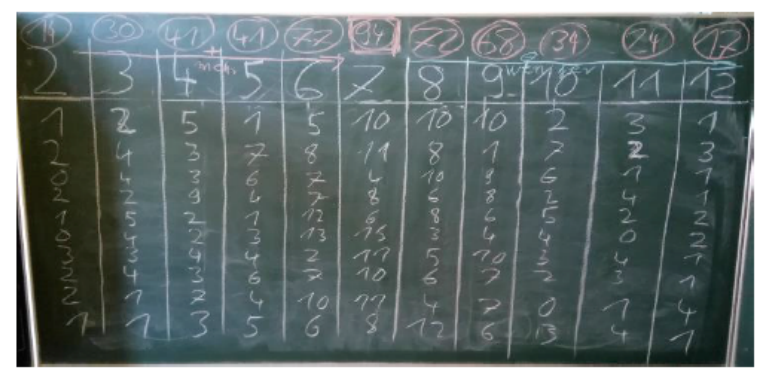

Figure 3: Documentation of results of the chance experiment ,throw of two dice“ of all ten groups on the board

Together with the teacher the pupils state that the most frequent appearing sum is " 7 " which appeared 94 of 500 times in this case, they also recognized that sums like "2" and "12" did not appear so often in comparison to the other sums. They also discussed some in their sense extreme experiences like group 9 who got the sum " 12 " four times or like group 2 who got the sum " 9 " only one time. The first insight for the pupils in lesson 5 is that the different sums of the throw of two dice are not equiprobable. This exploration is continued in lesson 6. In the sixth and seventh lesson the teacher reflects the results from the hands-on activity in the fifth lesson on the chance experiment "sum of two dice" and demonstrates how to set up this chance experiment in the TinkerPlots sampler (see Figure 4).
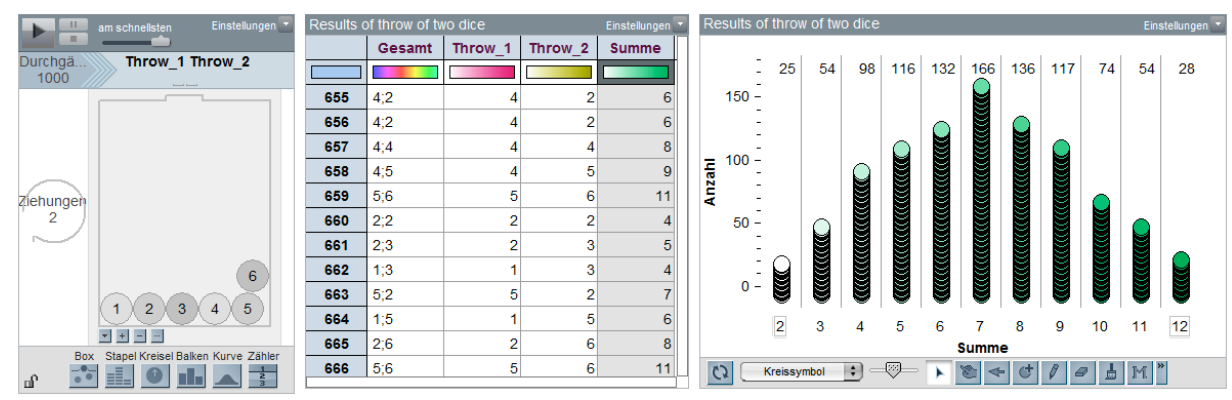

Figure 4: TinkerPlots sampler with results of the chance experiment „throw of two dice“

Accordingly he puts six balls into the box and labels them with the numbers 1-6. So this is a model for a fair dice. Then the teacher runs the sampler 1000 times, draws two balls from the box with replacement each time and creates a plot of the distribution of the sum of two dice. Together with the pupils the teacher discovers that the sum " 7 " showed up in 166 of 1000 cases (see Figure 4 right). The sum "6" has appeared 132 of 1000 times, the sum " 8 " 136 of 1000 times. Sums like "2" and "12" have appeared the fewest times (25 times of 1000, 28 times of 1000) compared to the other sums in this 
simulation. This is in line with the exploration they have already made in the hands-on-activity. The teacher repeats the simulation in TinkerPlots several times and the pupils observe that the mode of the distribution is the sum "7". Then the teacher asks the pupils for reasons why the sum "7" appears so frequently as the mode in the distributions and sums like " 3 " for example show up rather seldom. The pupils have the idea to consider the possible outcomes of the throw of two dice for each of the sums (2-12). In groups they identify the outcomes for each sum and collect their results on the board (see Figure 5). The pupils explore that the sum " 7 " has the most outcomes $(1+6,6+1,2+5,5+2,3+4,4+3)$ and therefore occurs more often in the experiment than for example the sum " 3 " (with the two outcomes $1+2$ and $2+1$ ) or the sum " 2 " (with the outcome $1+1$ ). The pupils also find out that there are 36 outcomes in total and each of them are equiprobable. So at the end the pupils together with the teacher make comparisons of probabilities of certain events like the sum " 6 " is more likely to show up than the sum " 3 " because there are five favorable out of 36 outcomes for sum " 6 " $(1+5,5+1,2+4$, $4+2,3+3)$ but only two favorable out of 36 outcomes for sum " 3 " $(1+2,2+1)$. This is also documented on the board in the classroom as we can see in Figure 5 and is the concluding result of the last lesson of the teaching unit. Further details about the design and the realization of the course can be read in Plückebaum (2018).

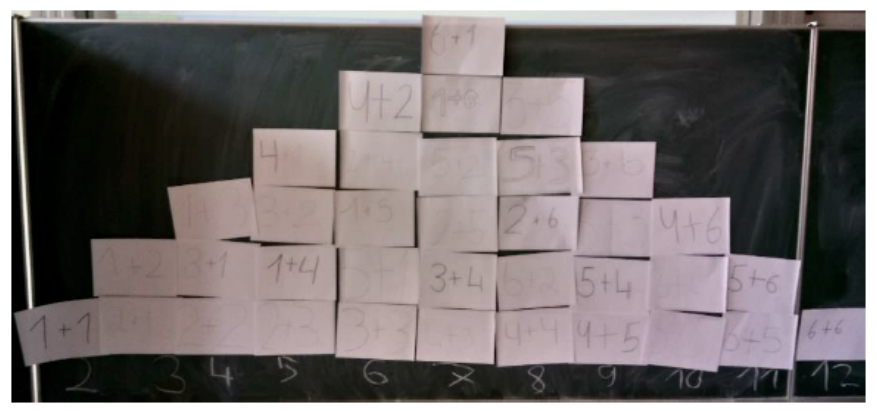

Figure 5: Documentation of possible outcomes of the experiment „sum of two dice“

\section{RESEARCH QUESTION}

The main aim of this paper was to design a teaching unit to develop primary school students reasoning about uncertainty. From an empirical point of view and for the evaluation of the teaching unit the following research question arises: In which way does the teaching unit develop the competence of pupils with regard to compare probabilities of events of multi-step chance experiments?

\section{DATA, PARTICIPANTS AND DATA ANALYSIS}

To supervise the learning processes of our pupils we collected data on different levels: (a) written pre/post-tests, (b) their working notes on tasks and activities after each lesson and (c) interviews after the teaching unit. The data were analyzed with qualitative methods. We will concentrate on (a) only. The written pre/post tests were handed out at the beginning and at the end of the teaching unit and they include six tasks. The posttest is identical to the pretest. In this paper we will only concentrate on the tasks which focus on the comparison of probabilities of events of multistep chance experiments (tasks 5 and 6 ).

Task (5) You and your friend Tim play a game where you toss a coin twice. You win if you get tail both times. Tim says that he wins if he gets tail and head when tossing the coin twice. Do you both have the same chances of winning? Explain!

Task (6) Anna and Tom are playing a game. They throw a dice twice. If the sum of the two dice is "7", Anna wins. If the sum is 3 or 12, Tom wins. Do Anna and Tom have the same chance of winning? Explain!

Both tasks tackle the issue of interpretation of probabilities of events in multi-step chance experiments. In task 5 the participants have to recognize that there are four equiprobable outcomes 
(HH, TT, HT, TH) and that Tim has a better chance of winning because he has two favourable cases (HT, TH) compared to the one favourable case TT of the other player. Accordingly the participants had to identify that in task 6 there are $(1+6,6+1,2+5,5+2,3+4,4+3)$ six favourable cases for the sum " 7 " compared to three favourable cases for the sums $3(1+2,2+1)$ and $12(6+6)$. Therefore the right conclusion in task 6 would be that Anna has a better chance to win. All in all 20 pupils of the teaching unit have participated in the pre- and posttest. We have collected the written pre- and posttests of the pupils and have analyzed them with qualitative methods.

\section{RESULTS}

After coding the correctness of the statements of each participant in pre-test and post-test, we did a frequency analysis and calculated the percentages of correct statements of each task in comparison to pre- and posttest. As we can see in Table 2, the primary school students' statistical reasoning skills with regard to comparing the probability of events of multi-step chance experiments have improved after the teaching unit compared to the situation before.

Table 2. Overview of results of pre and posttest for items 5 and 6

\begin{tabular}{llll}
\hline Item & \% pretest & \% posttest & Difference \\
\hline 5 & $00.00 \%$ & $47.38 \%$ & +47.38 \\
6 & $09.52 \%$ & $52.63 \%$ & +43.11 \\
\hline
\end{tabular}

We see that the percentage of correct answers in task 5 and 6 increases from pre- to posttest considerably. For task 5 there was no correct answer in the pretest, after the teaching unit nearly half of the participants $(47.38 \%)$ were able to solve task 5 correctly. For task 6 approx. $10 \%$ of the participants have given correct statements in the pretest, in the posttest more than half of the participants $(52.63 \%)$ were able to solve task 6 correctly. Let us have an exemplary look on two written excerpts for statements on task 5 to get an impression how the pupils have explained their statements. Toni provides a wrong statement in the pretest while stating that "Yes, we have an equal chance of winning." (see written note in German in Figure 6 left).
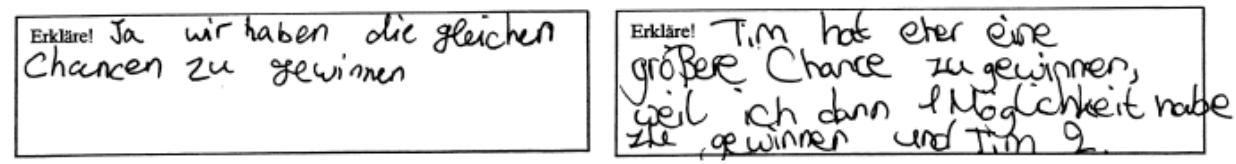

Figure 6: Note from the pre test of task 5 of Toni (left), Note from the post test of Tina (right)

This is a typical misconception that for example the outcomes $(\mathrm{H}, \mathrm{T})$ and $(\mathrm{T}, \mathrm{H})$ are not distinguished by Toni and he evaluates the chance of having tail twice and having tail and head as equiprobable. In the post test Tina recognises correctly that Tim has a better chance to win and provides the following correct statement: "Tim has a better chance to win because I have one chance to win and Tim has two." (see written note in German in Figure 6 right) Here Tina argues with the idea of Laplace: Tim has two favourable of four outcomes whence Tina has only one favourable of four outcomes. So that is why Tim is more likely to win the game. Let us also have a look at two selected statements in task 6. Alex states in the pretest incorrectly that "Tom will win because he has two numbers and has a better chance to win." (see written note in German in Figure 7 left). As a constrast example from the posttest Sarah concludes correctly that "Anna will win more often than Tom, because the 7 has more possibilities with two dice than the 3 and 12." (see written note in German in Figure 7 right) we might conclude that Sarah correctly identifies that the sum "7" has more favourable outcomes then the sums "3" and "12" together using the idea of the Laplace approach. 


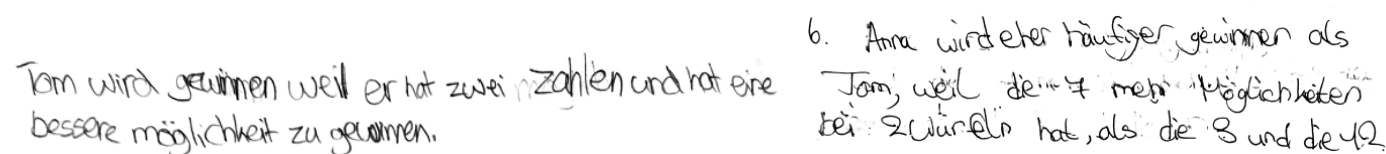

$$
\begin{aligned}
& \text { bessere möglichneit zu geoumen. }
\end{aligned}
$$

Figure 7: Note from the pre test of task 6 of Alex (left) and Note from the post test of task 6 of Sarah (right)

All in all we can say that the performance of the students from pre-test to post-test has increased considerably (see Table 2). Also the selected statements in the post-test show that the students' reasoning about the interpretation of events in multi-step chance experiments has developed in a positive way because the students are now able to use, e.g. the approach of Laplace to explain their statements when comparing the probability of events.

\section{SUMMARY \& OUTLOOK}

We have shown that a teaching unit taking into account all three conceptualizations of randomness (see Batanero, 2015) can be already implemented in primary school classroom. The results of the pre- and posttests show the considerable improvement of students' reasoning about the probability of events in multi-step chance experiments. In addition to that, we can say that the TinkerPlots sampler seems to be a powerful educational tool to facilitate the modelling and the data production process when simulating chance experiments in primary school. We can also implicate that the students should conduct the chance experiments as hand-on activities first and then use software like TinkerPlots for the modelling and data production process. Statistical reasoning should be initiated as early as possible, and the teaching unit and the study presented in this paper serve as one positive example how it can be done. Of course it remains a challenge to combine all three conceptualizations and to develop the reasoning of primary school students when interpreting and comparing probabilities in multi-step chance experiments. The teaching unit cannot only be of interest for primary school researchers and teachers but can also be adapted for implementation in secondary school classroom. The other data (working notes and interview data) collected in the frame of this research project are still under analysis.

\section{REFERENCES}

Batanero, C. (2015). Understanding Randomness: Challenges for Research and Teaching. In K. Krainer \& N. Vondrova (Eds.), Proceedings of the 9th Congress of the European Society for Research in Mathematics Education (pp. 34-49). Charles University in Prague: Faculty of Education and ERME.

Cobb, P., Confrey, J., diSessa, A., Lehrer, R., \& Schauble, L. (2003). Design Experiments in Educational Research. Educational Researcher, 32(1), 9-13.

Garfield, J., \& Ben-Zvi, D. (2008). Developing Students' Statistical Reasoning. Connecting Research and Teaching Practice. The Netherlands: Springer.

Hasemann, K., \& Mirwald, E. (2012). Daten, Häufigkeit Und Wahrscheinlichkeit. In G. Walther, M. van den Heuvel-Panhuizen, D. Granzer, \& O. Köller (Eds.), Bildungsstandards Für Die Grundschule: Mathematik Konkret (pp. 141-161). Berlin: Cornelsen Scriptor.

Jackes-Schulte, A. (2016). Design, Durchführung Und Evaluation Einer Unterrichtsreihe Zur Entwicklung Der Kompetenz "Gewinnchancen Beim Doppelten Würfelwurf Einzuschätzen" in Jahrgangsstufe 4. (Bachelor of Education), University of Paderborn.

Konold, C., \& Miller, C. (2011). Tinkerplots 2.0. Emeryville, CA: Key Curriculum Press.

Leavy, A., Meletiou-Mavrotheris, M., \& Paparistodemou, E. (2018). Statistics in Early Childhood and Primary Education: Supporting Early Statistical and Probabilistic Thinking. Singapore: Springer.

Plückebaum, K. (2018). Simulieren Von Zufallsexperimenten in Der Primarstufe Mit Der Software Tinkerplots. (Master of Education), Universität Paderborn, Paderborn.

Weustenfeld, W. (2007). Die Augensumme Zweier Würfel Voraussagen: Alles Nur Eine Frage Von Glück Oder Pech. Stochastik in der Schule, 27(3), 2-15. 